\title{
Detailed dynamic model for semiconductor optical amplifiers and their crosstalk and
} intermodulation distortion

\author{
Durhuus, Terji; Mikkelsen, Benny; Stubkjær, Kristian
}

Published in:

Journal of Lightwave Technology

Link to article, DOI:

$10.1109 / 50.156845$

Publication date:

1992

Document Version

Publisher's PDF, also known as Version of record

Link back to DTU Orbit

Citation $(A P A)$ :

Durhuus, T., Mikkelsen, B., \& Stubkjær, K. (1992). Detailed dynamic model for semiconductor optical amplifiers and their crosstalk and intermodulation distortion. Journal of Lightwave Technology, 10(8), 1056-1065. https://doi.org/10.1109/50.156845

\section{General rights}

Copyright and moral rights for the publications made accessible in the public portal are retained by the authors and/or other copyright owners and it is a condition of accessing publications that users recognise and abide by the legal requirements associated with these rights.

- Users may download and print one copy of any publication from the public portal for the purpose of private study or research.

- You may not further distribute the material or use it for any profit-making activity or commercial gain

- You may freely distribute the URL identifying the publication in the public portal 


\title{
Detailed Dynamic Model for Semiconductor Optical Amplifiers and Their Crosstalk and Intermodulation Distortion
}

\author{
Terji Durhuus, Benny Mikkelsen, and Kristian E. Stubkjaer, Member, IEEE
}

\begin{abstract}
An advanced dynamic model for multisection semiconductor optical amplifiers is presented. It accounts for the carrier and field distributions in the longitudinal direction as well as for the facet reflectivities. The model can handle arbitrary time-varying input signals and current modulations. Here the model is used to assess intermodulation distortion and crosstalk. First, cascaded amplifiers are considered, and the crosstalk and intermodulation distortion due to cascaded amplifiers are found to accumulate by adding together in amplitude; this may limit the number of cascaded amplifiers in multichannel systems. Carrierinduced nonlinearities depend strongly on facet reflectivities; for $25 \mathrm{~dB}$ of single-pass gain, a reflectivity of $5 \times 10^{-4}$ will result in 3-dB excess distortion. Reduction of intermodulation distortion by use of multisection amplifiers is found to be possible only for small channel separations $(<300 \mathrm{MHz})$. Simulations of 11-channel amplification showed a reduction of $13 \mathrm{~dB}$ in intermodulation distortion when random-phase optical carriers are applied.
\end{abstract}

\section{INTRODUCTION}

$\mathbf{F}$ UTURE high-capacity optical communication systems will employ high-density frequency division multiplexing (FDM) to fully utilize the available bandwidth and use optical amplifiers to compensate for transmission and splitting losses. Semiconductor optical amplifiers are promising candidates for this purpose due to their large bandwidth $(\sim 60 \mathrm{~nm}=8 \mathrm{THz})$ and low power consumption [1]-[4]. However, multichannel amplification can create system penalties due to crosstalk and intermodulation distortion (IMD) depending on the chosen modulation scheme and channel separation. Previous work related to this topic includes reports on two-channel crosstalk [5]-[8], gain suppression, and IMD [9]-[14]. Also, crosstalk degradation in a four-channel $565 \mathrm{Mb} / \mathrm{s}$ FSK heterodyne system has been reported [15].

This paper presents an advanced dynamic model for optical amplifiers that opposite to previous models [14], [16]-[19], accounts for the carrier density distribution in the longitudinal direction and for facet reflectivities, which influence carrierdensity-induced nonlinearities. Here we have restricted the use of the model to study intermodulation distortion and crosstalk, but the model can also be used to simulate absorptive and dispersive bistability as well as phase and amplitude

\footnotetext{
Manuscript received November 10, 1991; revised April 4, 1992. This work was supported under RACE Project 1027

The authors are with the Centre for Broadband Telecommunications, Electromagnetics Institute, Technical University of Denmark, DK-2800 Lyngby, Denmark.

IEEE Log Number 9201288.
}

modulation due to current modulation. Experimental measurements of intermodulation distortion and crosstalk, where nearly traveling-wave amplifiers are used, verify the validity of the model.

Section II describes a numerical model that can handle carrier-density-induced nonlinearities due to multichannel amplification. Section III presents a two-channel experimental setup with a self-heterodyne detection technique, which provides a large dynamic measurement range. Section IV gives the numerical and experimental results for crosstalk and intermodulation distortion in a two-channel system with two cascaded amplifiers. Also, the IMD reduction in multisection amplifiers as well as simulated IMD and channel output powers in an 11-channel system are considered.

\section{Numerical Model for SEmiconductor OPTICAL AMPLIFIER}

\section{A. Dynamic Equations}

In order to understand the carrier-induced nonlinearities in semiconductor optical amplifiers, a detailed numerical model is developed. The model is based on a traveling-wave description [18] of the electric field in the amplifier cavity, which is divided into sections each with a constant carrier density. By this sectioning, the model accounts for the longitudinal variations of both the electrical field and the carrier density. Previously developed models [5], [16]-[19] use an average value for the carrier density in the cavity; this is a good approximation for low input power levels. However, it is insufficient when the amplifier operates in saturation as is the case if the amplifier is used as a booster or a modulator, or if it is amplifying a large number of channels. Multisection amplifiers with inhomogenious current injection [20], [21] can also be simulated by the model, and it also offers the possibility of investigating multichannel amplification.

We consider the configuration depicted in Fig. 1 where the amplifier is divided into $n$ sections. Only the fundamental transverse mode is considered, and we account for the transverse modal structure by means of the effective index method, which reduces the problem to that of the propagation of a plane wave. The plane wave spectrum of the electric field $\hat{E}_{k, m}(t, z)$ can be written as [18]

$$
E_{k, m}(\omega, z)=\int_{-\infty}^{\infty} \hat{E}_{k, m}(t, z) e^{i \omega t} d t
$$




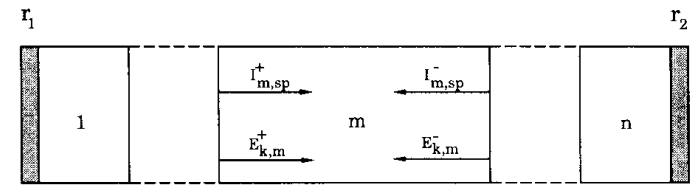

$z_{i}^{-} z_{m}^{+}$

Fig. 1. Schematic diagram of optical amplifier divided into $" 1$ sections. The electrical field due to the signal is denoted $E$ while the spontaneous intensity is given by $I$.

where $k$ and $m$ are the channel number and section number, respectively. The field $E_{k, m}(\omega, z)$ can be decomposed into right and left traveling fields as shown in Fig. 1.

$$
E_{k, m}(\omega, z)=E_{k, m}^{+}(\omega, z)+E_{k, m}^{-}(\omega \cdot z)
$$

where + and - indicate right- and left-traveling fields, respectively. The boundary conditions for section $m$ are given by

$$
\begin{aligned}
E_{k, m}^{+}\left(\omega, z_{m}^{-}\right) & =E_{k, m-1}\left(\omega, z_{m-1}^{+}\right) . \\
m & =2 \cdots n \\
E_{k, m}^{-}\left(\omega, z_{m}^{+}\right) & =E_{k, m+1}^{-}\left(\omega, z_{m+1}^{-}\right), \\
m & =1 \cdots n-1
\end{aligned}
$$

and for the special case where a $m$ equals 1 and $n$, the boundary conditions are

$$
\begin{array}{ll}
E_{k, 1}^{+}\left(\omega, z_{1}^{-}\right)=r_{1} E_{k, 1}^{-}\left(\omega, z_{1}^{-}\right)+E_{k}^{\mathrm{in}}(\omega), & m=1 \\
E_{k, n}^{-}\left(\omega, z_{n}^{+}\right)=r_{2} E_{k, n}^{+}\left(\omega, z_{n}^{+}\right) . & m=n
\end{array}
$$

where $r_{1}$ and $r_{2}$ are the field reflectivities at the left and right facet, respectively. The input field $E_{k}^{\text {in }}$ is taken at a reference plane immediately inside the left facet of the amplifier. In order to find the field evolution inside each section, we use the one-dimensional wave equation

$$
\frac{\partial^{2} E_{k, m}^{ \pm}(\omega, z)}{\partial z^{2}}+K_{m}^{2}\left(\omega, N_{m}\right) E_{k, m}^{ \pm}(\omega, z)=0
$$

which is known to have the solution

$$
E_{k, m}^{ \pm}(\omega, z)=E_{k, m}^{ \pm}\left(\omega, z_{m}^{\mp}\right) e^{\mp i K_{m}\left(\omega, \nu_{m}\right)\left(z-z_{m}^{\mp}\right)}
$$

if the wavenumber

$$
K_{m}\left(\omega, N_{m}\right)=\frac{\omega}{c} n_{e}\left(\omega, N_{m}\right)+i \frac{1}{2}\left(\Gamma g\left(\omega, N_{m}\right)-\alpha_{i}\right)
$$

is constant along the direction of propagation. In the model, we assume a constant carrier density $N_{m}$ and thereby a constant wavenumber in each section. $n_{e}\left(\omega, N_{m}\right)$ is the effective refractive index for the active layer in section $m, \Gamma$ is the confinement factor for the intensity of the fundamental waveguide mode, $\alpha_{i}$ is the internal loss per unit length, and $g\left(\omega, N_{m}\right)$ is the material gain given by [22]

$$
g\left(\omega, N_{m}\right)=a_{m}\left(N_{m}-N_{o}\right)-\gamma\left(\omega-\omega_{p}\left(N_{m}\right)\right)^{2}
$$

where $a_{m}$ and $\gamma$ are gain constants, $N_{o}$ is the carrier density at transparency, and $\omega_{p}$ is the peak wavelength, which is assumed to shift linearly with carrier density

$$
\omega_{p}\left(N_{m}\right)=\omega_{p o}+\frac{\partial \omega_{p}}{\partial N}\left(N_{n}-N_{r}\right)
$$

Here, $\omega_{p o}$ is the peak gain frequency at the carrier density $N_{r}$, and $\partial \omega_{p} / \partial N$ is taken to be constant. If we assume the signal frequencies to be appreciable only in a narrow band around the optical frequency $\omega_{k o}$, the slowly varying envelope approximation can be employed, and the wavenumber (9) can be replaced with its first-order Taylor expansion around $\omega_{k o}$

$$
K(\omega, N)=K\left(\omega_{k o}, N\right)+\frac{\partial K(\omega, N)}{\partial \omega}\left(\omega-\omega_{k o}\right)
$$

where

$$
\frac{\partial K(\omega \cdot N)}{\partial \omega}=\frac{n_{g}}{c}+i \frac{1}{2} \Gamma \frac{\partial g(\omega, N)}{\partial \omega}
$$

and the group index $n_{g}$ is given by

$$
n_{g}=n_{e}\left(\omega_{k o}, N\right)+\omega_{k o} \frac{\partial n_{e}(\omega, N)}{\partial \omega} .
$$

Equations (12)-(14) together with (8) give an expression for the field in the frequency domain at the output section $m$ if the input field to the section is known

$$
\begin{aligned}
E_{k, m}^{ \pm}\left(\omega, z_{m}^{ \pm}\right)= & E_{k, m}^{ \pm}\left(\omega, z_{m}^{\mp}\right) e^{-i K_{m}\left(\omega_{k o}, N_{m}\right) L_{m}} \\
& \cdot e^{-i \frac{n_{g}}{c}\left(\omega-\omega_{k_{o}}\right) L_{m}} \\
& \cdot e^{\frac{1}{2} \Gamma \frac{\partial g\left(\omega, N_{m}\right)}{\partial \omega^{2}}\left(\omega-\omega_{k_{o}}\right) L_{m}} .
\end{aligned}
$$

Here, $L_{m}$ denotes the length of section $m$. Next, we introduce the envelope functions of the electric fields at the border between the sections [18]

$$
F_{k, m}^{ \pm}\left(t, z_{m}^{ \pm}\right) e^{-i \omega_{k o} t}=\frac{1}{2 \pi} \int_{-\infty}^{\infty} E_{k, m}^{ \pm}\left(\omega, z_{m}^{ \pm}\right) e^{-i \omega t} d \omega .
$$

By this equation, we can transform (15) from the frequency to the time domain, where the electrical fields are described by the slowly varying envelope function $F_{k, m}^{ \pm}(t)$. Before applying (16) to (15), we introduce the following expansion of the last term in (15):

$$
e^{\frac{1}{2} \Gamma \frac{\partial g\left(\omega, N_{m}\right)}{\partial \omega}\left(\omega-\omega_{k o}\right) L_{m}} \approx 1-\frac{\Gamma}{2} i^{2} \frac{\partial g\left(\omega, N_{m}\right)}{\partial \omega}\left(\omega-\omega_{k o}\right) L_{m}
$$

where $\left(\omega-\omega_{k o}\right)$ will lead to a differentiation in the time domain while the term containing $e^{-i\left(\omega-\omega_{k_{0}}\right)}$ in (15) gives a time delay

$$
\begin{aligned}
F_{k, m}^{ \pm}\left(t, z_{m}^{ \pm}\right)= & e^{-i K_{m}\left(\omega_{k o}, N_{m}\right) L_{m}} \\
\cdot & {\left[1-i \frac{\Gamma}{2} \frac{\partial g(\omega, N)}{\partial \omega} L_{m} \frac{\partial}{\partial t}\right] } \\
\cdot & F_{k, m}^{ \pm}\left(t-\frac{n_{g}}{c} L_{m}, z_{m}^{\mp}\right) .
\end{aligned}
$$


Finally, the effective index is approximated to vary linearly with the carrier density and the real part of (9) is expanded around $\left(\omega_{p o}, N_{r}\right)$

$$
\begin{aligned}
\frac{\omega}{c} n_{e}(\omega, N) \approx & \frac{\omega_{p o}}{c} n_{e, o}+\frac{n_{g, o}}{c}\left(\omega-\omega_{p o}\right) \\
& +\frac{\omega_{p o}}{c} \frac{\partial n_{e}(\omega, N)}{\partial N}\left(N-N_{r}\right) .
\end{aligned}
$$

Here, $n_{e, o}$ and $n_{g, o}$ are the effective index and the group index, respectively, taken at the carrier density $N_{r}$ and at the angular frequency $\omega_{p o}$, and $\partial n_{e} / \partial N$ is assumed constant. In order to simplify the expression for the complex wavenumber, we introduce the following shorthand notations. The single-pass gain for a section

$$
G_{s, m}=e^{\left(\Gamma g\left(\omega_{k_{o}}, N_{m}\right)-\alpha_{i}\right) L_{m}}
$$

and the phase shift of the field in channel $k$ for the transit of section $m$.

$$
\begin{aligned}
\phi_{k, m}= & \frac{\omega_{p o}}{c} n_{e, o} L_{m}+\frac{n_{g, o}}{c}\left(\omega_{k o}-\omega_{p o}\right) L_{m} \\
& +\frac{\omega_{p o}}{c} \frac{\partial n_{e}(\omega, N)}{\partial N}\left(N-N_{r}\right) L_{m}
\end{aligned}
$$

Introducing (19)-(21) in (18) gives

$$
\begin{aligned}
F_{k, m}^{ \pm}\left(t, z_{m}^{ \pm}\right)= & \sqrt{G_{s, m}} e^{i \phi_{k, m}}\left[1-i \frac{\Gamma}{2} \frac{\partial g(\omega, N)}{\partial \omega} L_{m} \frac{\partial}{\partial t}\right] \\
& \cdot F_{k, m}^{ \pm}\left(t-\tau_{m}, z_{m}^{\mp}\right)
\end{aligned}
$$

where $\tau_{m}$ is the transit time for section $m$. Equation (22) together with the boundary conditions (3)-(6), which in the time domain can be expressed as

$$
\begin{gathered}
F_{k, m}^{+}\left(t, z_{m}^{-}\right)=F_{k, m-1}^{+}\left(t, z_{m-1}^{+}\right), \\
m \neq 1 \\
F_{k, m}^{-}\left(t, z_{m}^{+}\right)=F_{k, m+1}^{-}\left(t, z_{m+1}^{-}\right), \\
m \neq n \\
F_{k, 1}^{+}\left(t, z_{1}^{-}\right)=r_{1} F_{k, 1}^{-}\left(t, z_{1}^{-}\right)+F_{k}^{\text {in }}(t) e^{i\left(\omega_{p o}-\omega_{k o}\right) t}, \\
m=1 \\
F_{k, n}^{-}\left(t, z_{n}^{+}\right)=r_{2} F_{k, n}^{+}\left(t, z_{n}^{+}\right), \\
m=n
\end{gathered}
$$

form the backbone of the model. In the model, the facet reflectivities are assumed to be frequency independent. By solving (22)-(26) recursively in the time domain, ultra-highspeed dynamic properties of semiconductor optical amplifiers can be described. Time-varying phenomena with durations down to the order of a section transit time are the intrinsic limit for the validity of the model provided that the slowly varying envelope approximation is fulfilled.

To evaluate the carrier density $N_{m}$ in each section, the usual rate equation is employed

$$
\begin{aligned}
\frac{d N_{m}}{d t}=\frac{I_{m}}{e V_{m}}-R\left(N_{m}\right)- & {\left[g\left(\omega, N_{m}\right) S_{m, \text { sig }}\right.} \\
& \left.+a_{m}\left(N_{m}-N_{o}\right) S_{m, \text { spon }}\right] \frac{c}{n_{g, o}} .
\end{aligned}
$$

Here, $I_{m}$ is the bias current to the section, and $e$ and $V_{m}$ are the elementary charge and volume of the active region of section $m$, respectively. For the recombination rate $R(N)$, we use a detailed model [23]

$$
R(N)=c_{1} N+c_{2} N^{2}+c_{3} N^{3}
$$

where $c_{1}, c_{2}$, and $c_{3}$ are constants. The last term in (27) accounts for the stimulated emission where $S_{m \text {,sig }}$ and $S_{m \text {,spon }}$ are the average photon densities for the signal and spontaneous emission, respectively. $S_{m, \text { sig }}$ can be found if the slowly varying envelope functions $F_{k, m}^{ \pm}$are known at $z_{m}^{-}$and $z_{m}^{+}$, respectively [19]

$$
\begin{aligned}
S_{m, \text { sig }}= & \frac{\pi n_{g, o}}{L_{m} c \zeta} \int_{0}^{L_{m}}\left(\left|\sum_{k=1} \frac{1}{\sqrt{h \omega_{k}}} F_{m, k}^{+}\left(t, z_{m}^{--}\right)\right|^{2}\right) \\
& \cdot e^{\left(\Gamma g\left(\omega, N_{m}\right)-\alpha_{i}\right) z} d z \\
& +\frac{\pi n_{g, o}}{L_{m} c \zeta} \int_{0}^{L_{m}}\left(\left|\sum_{k=1} \frac{1}{\sqrt{h \omega_{k}}} F_{m, k}^{+}\left(t, z_{m}^{+}\right)\right|^{2}\right) \\
& \cdot e^{\left(\Gamma g\left(\omega, N_{m}\right)-\alpha_{i}\right) z} d z \\
& \frac{G_{s, m}-1}{\ln \left(G_{s, m}\right)} \frac{\pi n_{g, o}}{c \zeta} \\
& \cdot\left(\left|\sum_{k=1} \frac{1}{\sqrt{h \omega_{k}}} F_{m, k}^{+}\left(t, z_{m}^{-}\right)\right|^{2}\right. \\
& \left.+\left|\sum_{k=1} \frac{1}{\sqrt{h \omega_{k}}} F_{m, k}^{-}\left(t, z_{m}^{+}\right)\right|^{2}\right) .
\end{aligned}
$$

Here, $h$ is Planck's constant, and $\zeta=\sqrt{\mu_{o} / \varepsilon_{o}} / n_{e, o}$ is the wave impedance where $\varepsilon_{0}$ is the vacuum permittivity and $\mu_{o}$ is the vacuum permeability. In order to find the average photon density originating from the spontaneous emission $S_{m, \text { spon }}$, we use the following expression for the variation of the spontaneous intensity in the $z$-direction [19]:

$$
\begin{aligned}
\frac{d I_{m, \mathrm{sp}}^{ \pm}(\omega, z)}{d z}= & \pm\left[\Gamma g\left(\omega, N_{m}\right)-\alpha_{i}\right] I_{m, \mathrm{sp}}^{ \pm}(\omega, z) \\
& \pm \frac{\beta(\omega) R_{r}\left(N_{m}\right) h \omega}{4 \pi}
\end{aligned}
$$

where $\beta(\omega)$ is the fraction of spontaneous emission at the frequency $\omega$ coupled into the traveling waves, and $R_{r}\left(N_{m}\right)=$ $c_{2} N_{m}^{2}$ is the recombination rate due to radiative recombinations. Equation (30) has the following solution when the gain is assumed constant in each section:

$$
\begin{aligned}
I_{m, \mathrm{sp}}^{ \pm}(\omega, z)= & \frac{\beta(\omega) R_{r}\left(N_{m}\right) h \omega}{4 \pi\left(\Gamma g\left(\omega, N_{m}\right)-\alpha_{i}\right)} \\
& \cdot\left[C^{ \pm} e^{ \pm\left(\Gamma g\left(\omega, N_{m}\right)-\alpha_{i}\right)\left(z-z_{m}^{\mp}\right)}-1\right] .
\end{aligned}
$$

The integration constants $C^{ \pm}$can be found if the spontaneous intensities are known at the intersection between the sections

$$
C^{ \pm}=I_{m, \mathrm{sp}}^{ \pm}\left(\omega, z_{m}^{\mp}\right) \frac{4 \pi\left(\Gamma g\left(\omega, N_{m}\right)-\alpha_{i}\right)}{\beta(\omega) R_{r}\left(N_{m}\right) h \omega}+1,
$$


In the same way as for the signal field, the boundary conditions for the spontaneous intensities can be written as

$$
\begin{aligned}
I_{m, \mathrm{sp}}^{+}\left(\omega, z_{m}^{-}\right) & =I_{m-1, \mathrm{sp}}^{+}\left(\omega, z_{m-1}^{+}\right), & & m \neq 1 \\
I_{m, \mathrm{sp}}^{-}\left(\omega, z_{m}^{+}\right) & =I_{m+1, \mathrm{sp}}^{-}\left(\omega, z_{m+1}^{-}\right), & & m \neq n \\
I_{1, \mathrm{sp}}^{+}\left(\omega, z_{1}^{-}\right) & =r_{1}^{2} I_{1, \mathrm{sp}}^{-}\left(\omega, z_{1}^{-}\right), & & m=1 \\
I_{n, \mathrm{sp}}^{-}\left(\omega, z_{n}^{+}\right) & =r_{2}^{2} I_{n, \mathrm{sp}}^{+}\left(\omega, z_{n}^{+}\right), & & m=n .
\end{aligned}
$$

The average spontaneous photon density in each section is calculated as

$$
\begin{aligned}
S_{m, \mathrm{sp}}= & \frac{4 \pi n_{g, o}}{h \omega_{p 0} c} \frac{1}{L_{m}}\left[\int_{z_{m}^{-}}^{z_{m}^{+}} I_{m, \mathrm{sp}}^{+}(\omega, z) d z\right. \\
& \left.+\int_{z_{m}^{+}}^{z_{m}^{-}} I_{m, \mathrm{sp}}^{-}(\omega, z) d z\right]
\end{aligned}
$$

and inserting (31) and (32) in (37) gives

$$
\begin{gathered}
S_{m, \mathrm{sp}}=\frac{4 \pi n_{g . o}}{h \omega_{p 0} c}\left[\left(I_{m, \mathrm{sp}}^{+}\left(z_{m}^{-}\right)+I_{m, \mathrm{sp}}^{-}\left(z_{m}^{+}\right)\right.\right. \\
\left.+\frac{\beta R_{r}\left(N_{m}\right) h \omega_{p 0}}{2 \pi\left(\Gamma g\left(\omega_{p 0}, N_{m}\right)-\alpha_{i}\right)}\right) \\
\left.\cdot\left(\frac{G_{s, m}-1}{\ln G_{s, m}}\right)\right] \\
-\frac{2 \beta R_{r}\left(N_{m}\right) n_{g, o}}{\left(\Gamma g\left(\omega_{p 0}, N_{m}\right)-\alpha_{i}\right) c}
\end{gathered}
$$

where the effective spontaneous emission factor $\beta=\sum_{i} \beta\left(\omega_{i}\right)$ is introduced [19] and $\omega_{i}$ is a resonance frequency for the amplifier cavity. In the conversion from spontaneous intensity to photon density, an average photon energy of $h \omega_{p o} / 2 \pi$ is used.

The signal, the spontaneous intensity, and the carrier density can now be found in the time domain in each section by numerical solution of (23)-(27) and (33)-(36).

In order to express the output power of the amplifier in terms of the slowly varying envelope function, we use [5]

$$
P^{o}(t)=\frac{\left(1-r_{2}^{2}\right) \sum_{k=1}\left|F_{n, k}^{+}\left(t, z_{n}^{+}\right)\right|^{2} W d}{2 \zeta \Gamma}
$$

and the coupled input power in channel $k$ is expressed by

$$
P_{k}^{\text {in }}(t)=\frac{\left|F_{k}^{\mathrm{in}}(t)\right|^{2} W d}{2 \zeta\left(1-r_{1}^{2}\right) \Gamma} .
$$

An example of the carrier density along the cavity and the signal power distribution for the field traveling in the positive and negative $z$-direction is given in Fig. 2 for an amplifier divided into 10 sections. The input power and gain are $-23 \mathrm{dBm}$ and $25 \mathrm{~dB}$, respectively, and the facet reflectivities are $10^{-3}$. The dips in the carrier density are due to the high signal field and spontaneous intensity at the facets. This model can be used to study ultrafast dynamical phenomena ( $\sim 1 \mathrm{ps})$ in semiconductor optical amplifiers, but in the following, we use the model to study intermodulation distortion and crosstalk effects that will cause time variations in the order of $1 \mathrm{~ns}$.

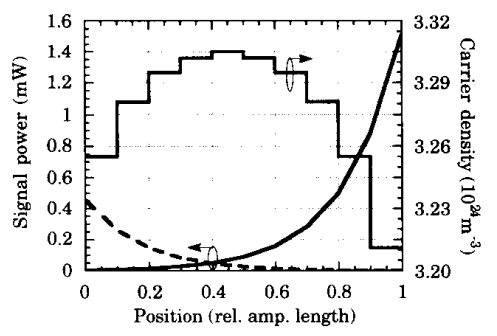

Fig. 2. Signal power for the right and left traveling field and carrier density along the amplifier cavity. Gain: $25 \mathrm{~dB}$; input power: $-23 \mathrm{dBm}$; reflectivity: $10^{-3}$. Solid curve: forward traveling field. Dashed curve: backward traveling field.

\section{B. Definition of IMD and Crosstalk}

From (29) we see that the photon density $S_{m, \text { sig }}$ associated with the signal is proportional to

$$
\left|\sum_{k=-1} \frac{1}{\sqrt{h \omega_{k o}}} F_{m, k}^{ \pm}\left(t, z_{m}^{\mp}\right)\right|^{2} \text {. }
$$

This will cause a time-dependent variation of the carrier density corresponding to the beat frequency between the channels. The modulation of the carrier density generates a modulation of the gain and the refractive index; this again leads to an amplitude and phase modulation of the channels. If, for convenience, we consider only two input channels, the slowly varying functions (25) at the input can be written as

$$
\begin{aligned}
& F_{1}^{\mathrm{in}}(t) e^{i\left(\omega_{\mathrm{po}}-\omega_{1 o}\right) t}=\hat{F}_{1}^{\mathrm{in}} e^{i(\Delta \omega) t} \\
& F_{2}^{\mathrm{in}}(t) e^{i\left(\omega_{p o}-\omega_{2 o}\right) t}=\hat{F}_{2}^{\mathrm{in}} e^{i(\Delta \omega+\Omega) t}
\end{aligned}
$$

where the envelope function $\hat{F}^{\text {in }}$ is constant, and the channel spacing is denoted by $\Omega$. At the output of the amplifier, the signal $\hat{F}^{o}(t)$ consists of the two amplified input signals plus a number of intermodulation distortion products (IMD) with the amplitude $\hat{F}_{\text {IMD, } p}$

$$
\begin{aligned}
F^{o}(t)= & \hat{F}_{1}^{o} e^{i(\Delta \omega) t}+\hat{F}_{2}^{o} e^{i(\Delta \omega+\Omega) t} \\
& +\sum_{p=-\infty}^{\infty} \hat{F}_{\mathrm{IMD}, p} e^{i[\Delta \omega+p \Omega] t} .
\end{aligned}
$$

The relative third-order intermodulation distortion at the frequencies $2 f_{1 o}-f_{2 o}$ are now defined as

$$
\begin{aligned}
& \frac{P_{\mathrm{IMD} 1}}{P_{1}^{o}}=\frac{\left|\hat{F}_{\mathrm{IMD},-1}\right|^{2}}{\left|\hat{F}_{1}^{o}\right|^{2}} \\
& \frac{P_{\mathrm{IMD} 2}}{P_{2}^{o}}=\frac{\left|\hat{F}_{\mathrm{IMD}, 2}\right|^{2}}{\left|\hat{F}_{2}^{o}\right|^{2}}
\end{aligned}
$$

where $f_{i}=\omega_{i} / 2 \pi$.

The origin of crosstalk in semiconductor optical amplifiers is also due to carrier density modulations. An AM-modulated channel will modulate the carrier density due to the variation in $S_{m, \mathrm{sig}}$ (27), and thereby also the gain experienced by the other channels. The origin of FM crosstalk is somewhat 
TABLE I

List of Parameter Values

\begin{tabular}{|c|c|c|}
\hline Symbol & Parameter & Value \\
\hline$a_{m}$ & gain factor & $1.75 \times 10^{-20} \mathrm{~m}^{2}\left(N_{m}>N_{o}\right)$ \\
\hline$c_{1}$ & recombination coefficient & $1.5 \times 10^{8} \mathrm{~s}^{-1}$ \\
\hline$c_{2}$ & recombination coefficient & $2.5 \times 10^{-17} \mathrm{~m}^{3} \mathrm{~s}^{-1}$ \\
\hline$c_{3}$ & recombination coefficient & $9.4 \times 10^{-41} \mathrm{~m}^{3} \mathrm{~s}^{-1}$ \\
\hline$d$ & active layer thickness & $0.15 \mu \mathrm{m}$ \\
\hline$L$ & amplifier length & $500 \mu \mathrm{m}$ \\
\hline$L_{m}$ & section length & $50 \mu \mathrm{m}(n=10)$ \\
\hline$n$ & number of sections & 10 \\
\hline$n_{\epsilon, o}$ & refractive index & 3.5 \\
\hline$n_{g, O}$ & group index & 3.75 \\
\hline$N_{o}$ & carrier density at transparency & $0.9 \times 10^{24} \mathrm{~m}^{-3}$ \\
\hline$N_{r}$ & reference carrier density & $3 \times 10^{24} \mathrm{~m}^{-3}$ \\
\hline$r_{1}^{2}$ & residual facet reflectivity & $5 \times 10^{-5}$ \\
\hline$r_{2}^{1}$ & residual facet reflectivity & $5 \times 10^{-5}$ \\
\hline$u^{2}$ & active region width & $3.3 \mu \mathrm{m}$ \\
\hline$\alpha_{i}$ & internal loss & $1500 \mathrm{~m}^{-1}$ \\
\hline$\beta$ & spontaneous emission factor & $5 \times 10^{-5}$ \\
\hline$\partial n_{\epsilon} / \partial N$ & refractive index shift coefficient & $-1.33 \times 10^{-26} \mathrm{~m}^{-3}$ \\
\hline$\partial \omega_{p} / \partial N$ & frequency shift coefficient & $2.12 \times 10^{-11} \mathrm{~m}^{3} \mathrm{~s}^{-1}$ \\
\hline$\gamma$ & gain factor & $2.7 \times 10^{-24} \mathrm{~m}^{-1} \mathrm{~s}^{-2}$ \\
\hline$\Gamma$ & confinement factor & 0.31 \\
\hline$\omega_{p o}$ & gain peak frequency & $1.24 \times 10^{15} \mathrm{rad} / \mathrm{s}$ \\
\hline
\end{tabular}

more complicated. An FM-modulated signal will lead to an intensity modulation because of the discriminator effect of the Fabry-Perot ripple caused by the facet reflectivities. The intensity variation will modulate the carrier density and thereby the refractive index (19) that results in FM crosstalk. A second mechanism for FM crosstalk is the modulation of the frequency separation between the channels and thus the gain suppression effects [9]. However, FM crosstalk due to the latter effect is less than $-45 \mathrm{~dB}$ for input powers below the saturation input power.

In the definition of crosstalk it is necessary to distinguish between pure AM or FM crosstalk and the case of a mixture of AM and FM crosstalk. For pure AM or FM modulation, the crosstalk is defined in the same way as in [5]. However, in practical experiments it is impossible to have pure frequency modulation because of spurious AM modulation. The crosstalk will consequently be a mixture of FM and AM crosstalk, and therefore a third definition of crosstalk is needed. If we assume that channel 1 is operating $\mathrm{CW}$ and channel 2 is modulated, the output from the amplifier can be written as

$$
P_{l}^{o}(t)=\hat{P}_{l, o}^{o}+\sum_{p=1}^{\infty} \hat{P}_{l, o}^{o} \sin \left(p \omega_{m} t+\phi_{l, p}\right), \quad l=1,2
$$

where $\hat{P}_{l, o}^{o}$ is the power of the different frequency components. In this case, we define the crosstalk $\gamma$ as the normalized power of the sidebands in channel 1 divided by the normalized power of the sidebands in channel 2 .

$$
\gamma=\left[\frac{\sum_{p=1}^{\infty} \hat{P}_{1, p}^{o}}{\hat{P}_{1, o}^{o}+\sum_{p=1}^{\infty} \hat{P}_{1, p}^{o}}\right] /\left[\frac{\sum_{p=1}^{\infty} \hat{P}_{2, p}^{o}}{\hat{P}_{2, o}^{o}+\sum_{p=1}^{\infty} \hat{P}_{2, p}^{o}}\right] .
$$

The parameters for the amplifier used in the numerical simulations are given in Table I.

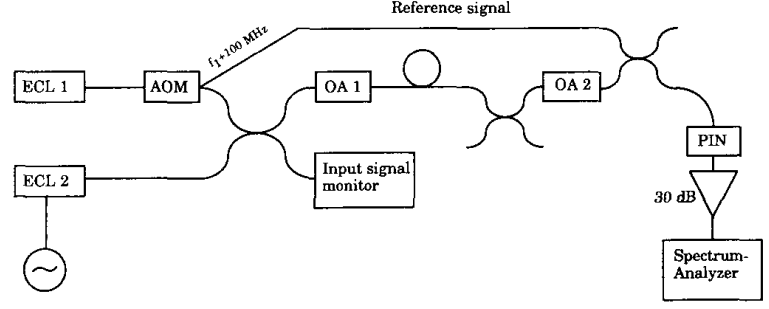

Fig. 3. Two-channel self-heterodyne experimental setup.

\section{Two-Channel ExPerimental Setup}

In order to verify the theoretical model, a two-channel selfheterodyne setup was built. This detection technique offers a dynamic range of more than $50 \mathrm{~dB}$ and consequently, allows for precise measurement of IMD and crosstalk for even small input power levels [13]. A schematic of the setup is shown in Fig. 3. The single-mode laser sources consist of external cavity lasers (ECL's) that can be directly modulated. By means of an acoustooptic modulator, a $100-\mathrm{MHz}$ frequency shifted signal, used as the reference signal for the heterodyne detection, is extracted from channel 1 . The two signals-channel 1 and 2 -are directed to the optical amplifier (OA1) via a fiber coupler. The other branch of the coupler provides samples of the combined signals for a Fabry-Perot interferometer, a power meter, and a spectrometer. The output signal from the amplifier (OA2) is combined with the reference signal in a fiber coupler and detected by a PIN photodetector with $25-\mathrm{GHz}$ bandwidth. The detector output is amplified to $30 \mathrm{~dB}$, and the down converted spectrum can be observed with a microwave spectrum analyzer.

The optical amplifiers used in the experiments are ARcoated $7^{\circ}$ angled facet InGaAsP amplifiers based on the Ridge waveguide structure [3]. For a bias current of $150-200 \mathrm{~mA}$, 


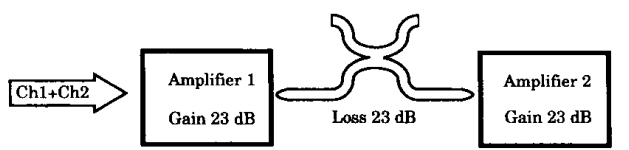

Fig. 4. Schematic of the cascaded amplifiers.

the internal gain for the TE mode is typically $25 \mathrm{~dB}$ with a ripple of $0.3 \mathrm{~dB}$, corresponding to a facet reflectivity of $5 \times 10^{-5}$. The peak gain wavelength is $1515 \mathrm{~nm}$, the optical bandwidth is $60 \mathrm{~nm}$, and the $3 \mathrm{~dB}$ saturation input power is typically $-17 \mathrm{dBm}$.

\section{RESULTS}

In this section, we use the model from Section II to predict the crosstalk and intermodulation distortion (IMD) for different amplifier configurations. The results lead to conclusions about the preferred operating conditions. For two-channel configurations, the results of the model are compared with measurements to verify the accuracy of the model.

\section{A. Two-channel Crosstalk and IMD in Cascaded Amplifiers}

In [25] it is predicted that many amplifiers can be cascaded if only the accumulation of the spontaneous emission is taken into account. The spontaneous emission from each amplifier stage is added in power because of the incoherence of the spontaneous light. In the following we will investigate how crosstalk and intermodulation distortion increases in systems containing cascaded amplifiers.

In the experiments, two semiconductor optical amplifiers are cascaded as shown in Fig. 4 to measure the distortion after the first and second amplifiers. Both are operating at a gain of $23 \mathrm{~dB}$, and the total loss between them is adjusted to $23 \mathrm{~dB}$ to ensure similar input power levels.

For the crosstalk measurements, laser 1 (channel 1) is operated $\mathrm{CW}$ while laser 2 is modulated. The direct modulation of the external cavity lasers results in a mixture of AM and FM modulation, and consequently the observed crosstalk will be a mixture of AM and FM crosstalk.

Fig. 5 shows the crosstalk levels as a function of input power in channel 2 while the input power in channel 1 is held constant at $-22.8 \mathrm{dBm}$. The channel spacing is $400 \mathrm{MHz}$, and the $30-\mathrm{MHz}$ modulation of channel 2 results in $\mathrm{AM}$ and FM indices of 0.2 and 0.3 , respectively. In Fig. 5, triangles and squares give the measured crosstalk level after the first and second amplifier while the solid lines give the theoretical predictions obtained by the numerical model. For low input power, the increase in crosstalk level is approximately $6 \mathrm{~dB}$ after the second amplifier, indicating that the contributions from each amplifier stage are added in amplitude (and not in power). The observed crosstalk is relatively high because of the spurious AM modulation of the signal in channel 2 [5]. For pure FM modulation, the model predicts that the crosstalk would be approximately $40 \mathrm{~dB}$ lower. If a $1.0-\mathrm{dB}$ power penalty due to crosstalk can be tolerated, the crosstalk should be lower than $-17 \mathrm{~dB}$ [24]. According to Fig. 5, this corresponds to an upper limit for the input power in the modulated channel of $-28 \mathrm{dBm}$ for the cascaded amplifiers and $-25 \mathrm{dBm}$ for a single amplifier.

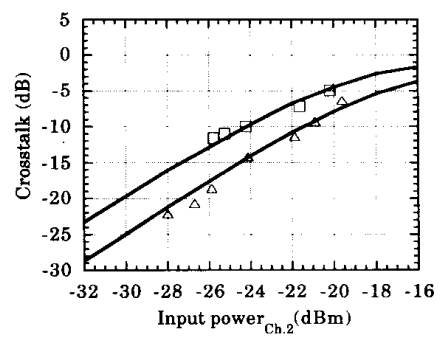

Fig. 5. Crosstalk versus input power to channel 2 after the first triangles and second squares amplifiers. Curves slow theoretical results. AM index: 0.2 ; FM index: 0.3 ; input power to channel $1:-22.8 \mathrm{dBm}$; channel spacing: $400 \mathrm{MHz}$; modulation frequency: $30 \mathrm{MHz}$.

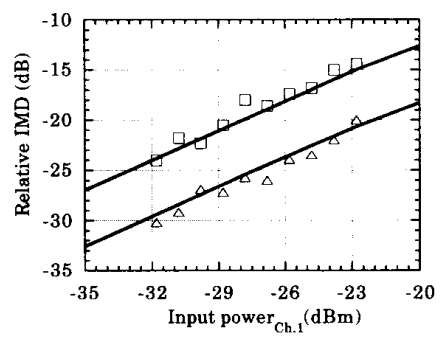

Fig. 6. Relative IMD $\left(2 f_{1 o}-f_{2 o}\right)$ versus input power to channel 1 after the first (triangles) and second (squares) amplifier. Curves show theoretical results. Input power to channel 2 : $-19.5 \mathrm{dBm}$; channel spacing: $400 \mathrm{MHz}$.

The relative third-order IMD of the configuration in Fig. 4 is also investigated as a function of the input power in channel 1 as shown in Fig. 6. Both channels are operated $\mathrm{CW}$ with a spacing of $400 \mathrm{MHz}$, and the input power in channel 2 is kept constant at $-19.5 \mathrm{dBm}$. As seen, the relative IMD, defined by (44), is $6 \mathrm{~dB}$ higher after the second amplifier stage compared to the first stage. The model predictions given by the solid lines are in good agreement with the experimental results. As also predicted by small-signal analysis [25], the IMD at $2 f_{1 o}-f_{2 o}$ is proportional to $\left(P_{1}^{\text {in }}\right)^{2} P_{2}^{\text {in }}$ so small input power levels are preferable. On the other hand, the signal-to-noise ratio will degrade for lower input power levels due to dominating signalspontaneous beat noise [25]; consequently, there is a trade-off between low IMD and low noise.

A low IMD can also be obtained by increasing the channel spacing as shown in Fig. 7 where the relative IMD is given for $25-\mathrm{dB}$ gain and an input power of $-23 \mathrm{dBm}$ per channel. The IMD depends on the channel spacing $\Delta f$, due to the finite bandwidth of the carrier response, which is proportional to $1 /\left[1+4 \pi^{2} \Delta f^{2} \tau_{e} 2\right]$ [26]. $\tau_{e}$ is the effective carrier lifetime, which in section $m$ of the amplifier can be found from [30]

$$
\begin{aligned}
\tau_{e, m}^{-1}= & \frac{d R\left(N_{m}\right)}{d N}+ \\
& \frac{d}{d N}\left[g\left(\omega, N_{m}\right) S_{m, \text { sig }}+a_{m}\left(N_{m}-N_{o}\right) S_{m, \text { spon }}\right] \\
& \cdot \frac{c}{n_{g, o}}
\end{aligned}
$$




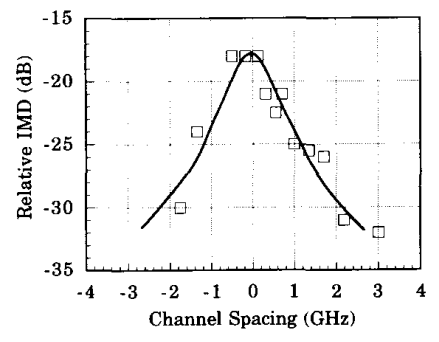

Fig. 7. Relative IMD $\left(2 f_{1 o}-f_{2 o}\right)$ versus channel spacing. Gain: $25 \mathrm{~dB}$; input power: $-23 \mathrm{dBm} /$ channel.

As an example, the IMD decreases approximately $10 \mathrm{~dB}$ when the channel spacing is increased from $400 \mathrm{MHz}$ to $2 \mathrm{GHz}$. The results are for a single amplifier stage, but the relative shape of the curve will remain the same for cascaded amplifiers. Note that different polarizations for the channels would also reduce the IMD.

The result for cascaded amplifiers shows that the amplitudes of the crosstalk and IMD contributed by each amplifier stage are added together and therefore accumulate as $20 \log _{10} N$ (in decibels) in a link with $N$ amplifier stages. This is faster than the accumulation of spontaneous noise for which the power from each stage is addled (i.e., $10 \log _{10} N$ ). Consequently, crosstalk and IMD will be the factors that limit the number of cascaded amplifiers in a multichannel system. Since the relative IMD is proportional to the gain in the two channels [26] and the crosstalk is proportional to the gain in the disturbing channel, it is generally advantageous to use $N$ amplifier stages with a smaller gain for a given required link gain. The optimum $N$ is determined by the trade-off between crosstalk and IMD reduction due to smaller gain and by the accumulation given by $20 \log _{10} N$.

The results presented above are for amplifiers with small facet reflectivities $\left(5 \times 10^{-5}\right)$. The reflected fields as described by (24) and (26) contribute significantly to the IMD. As an example, our model predicts that, for $25-\mathrm{dB}$ gain and for facet reflectivities of $5 \times 10^{-3}$ and $5 \times 10^{-4}$, the excess IMD will be approximately 6 and $3 \mathrm{~dB}$ compared to a traveling-wave amplifier [31]. For a reflectivity of $5 \times 10^{-5}$, the excess IMD is less than $0.5 \mathrm{~dB}$ so clearly reflectivities of that order are desirable. Several types of amplifiers meet this requirement. For example, angled-facet amplifiers have achieved reflectivities of $1-5 \times 10^{-5}$ [3]. Similarly, straight facet amplifiers with double-layer AR coats [2] and window structure amplifiers [27] have achieved reflectivities of about $10^{4}$

\section{B. Reduction of IMD by Inhomogenious Carrier Injection}

Several suggestions have been given for reduction of the IMD by external means, but these approaches are complex and difficult to implement in practical systems [13], [28]. Recently, IMD measurements on a three-section amplifier showed a decrease in IMD levels for certain operating conditions [20]. Following these results we have studied a similar amplifier configuration with one absorber section between two gain sections as depicted in Fig. 8. Three signals are injected to the

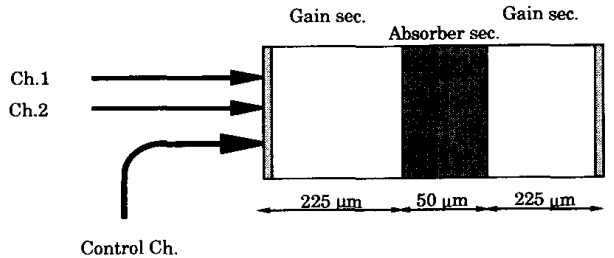

Fig. 8. Schematic of optical amplifier with an absorber section between two gain sections.

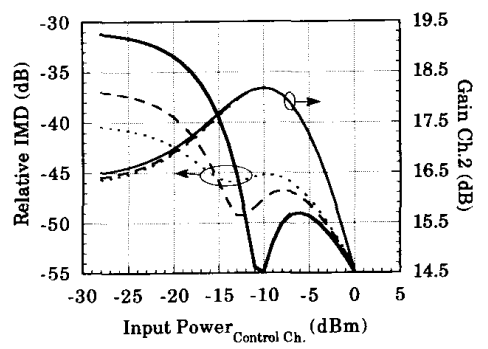

Fig. 9. Relative IMD and gain versus input power to the control channel. Signal input power: $-25 \mathrm{dBm} /$ channel; channel spacing $32 \mathrm{MHz}$; $\partial n_{e} / \partial N$ in absorber section: $-1.33 \times 10^{-26} \mathrm{~m}^{-3}$ (dotted curves), $-2.66 \times 10^{-26} \mathrm{~m}^{-3}$ (dashed curves), $-5.32 \times 10^{-26} \mathrm{~m}^{-3}$.

amplifier; two of them are ordinary signals close in frequency while the third is a control signal that adjusts the absorption in the center section. The control channel is separated $30 \mathrm{~nm}$ from the signal channels so beating phenomena between the signal and the control channel can be neglected. Fig. 9 shows the relative IMD and gain in channel 2 as a function of the input power in the control channel. The results are shown for three different values of $d n_{e} / d N$ in the absorber section. At low carrier densities, $\left|d n_{e} / d N\right|$ increases so we have chosen the same value and two and four times the value of $d n_{e} / d N$ in the gain sections. The differential gain is also higher in the absorber section [29], and in these simulations a value four times that of the gain sections is assumed. The bias currents to the absorber $(50 \mu \mathrm{m})$ and gain $(225 \mu \mathrm{m})$ sections are 0.5 and $180 \mathrm{~A}$, respectively. The input power in each channel is $-25 \mathrm{dBm}$, and the channel spacing is $32 \mathrm{MHz}$. When the control signal is increased, the relative IMD decreases to a minimum value that occurs at input powers between -15 and $-10 \mathrm{dBm}$. This reduction of IMD is caused by the carrier-induced nonlinearities in the absorber section, which are in counterphase with those in the gain sections. From (27) it can be seen that the last term, accounting for the stimulated emission, will be positive for carrier densities above transparency and negative for carrier densities below transparency (absorption). The phase and amplitude modulation of the signal in the gain and absorber section will consequently be in counterphase and for specific operating conditions cancel at the output of the amplifier. The largest reduction in IMD (25 dB) occurs for the largest value of $\left|d n_{e} / d N\right|$. Also the gain is increasing with input power because of the decrease in the absorption caused by the optical pumping of the center section. For an input power larger than $-10 \mathrm{dBm}$, the saturation of the gain sections becomes dominant and the total gain decreases. 


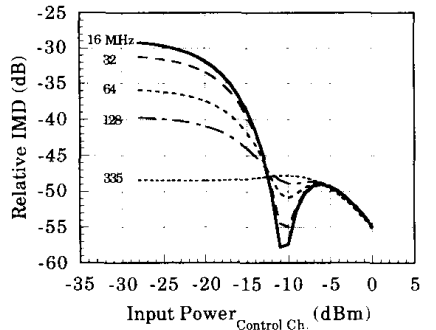

Fig. 10. Relative IMD versus input power to control channel for different channel spacings. Signal input power: $-2.5 \mathrm{dBm} /$ channel; $\partial n_{\epsilon} / \partial N^{\circ}$ in absorber section: $-5.32 \times 10^{-26}$.

The carrier-induced nonlinearities that generate IMD have a low-pass characteristic that depends on the effective carrier lifetime. For low photon densities, the first term in (48) is dominant, and from (28) it is seen that $\tau_{e}$ in the absorber section is considerably longer than in the gain sections. This means that the compensation of IMD by the absorber section is limited to small channel separations. Fig. 10 shows the relative IMD as a function of input power in the control channel for different channel separations. The largest reduction in IMD is obtained for the lowest channel spacing, and at a channel spacing of $335 \mathrm{MHz}$ the absorber section cannot reduce the IMD. For input powers larger than $-7 \mathrm{dBm}$, the absorber is saturated $\left(N_{m}=N_{o}\right)$ and the relative IMD is almost equal for different channel spacings. This can be explained by the large bandwidth of the carrier response in the gain sections governed by the second term in (48) at high photon densities.

The results given in Fig. 10 clearly show that the reduction of IMD in multisection amplifiers is limited to low channel separations $(<300 \mathrm{MHz})$ due to the small bandwidth of the carrier density dynamics in the absorber section.

\section{Multichannel Amplifications}

There are mainly two reasons for considering channels that are so densely spaced that IMD will influence the performance of a system: 1) In future optical high-capacity distribution networks the amplifier bandwidth cannot always be viewed as an abundant resource. 2) The requirements to tuneable local oscillators or tuneable filters are more relaxed if the occupied bandwidth and thereby the tuning range is small. In the calculations, we simulate a system containing 11 channels. The input power in each channel is $-30 \mathrm{dBm}$, and the single pass gain is $25 \mathrm{~dB}$. In Fig. 11 the output signal and IMD are given in each channel for a channel separation of (a) $300 \mathrm{MHz}$, (b) $3.3 \mathrm{GHz}$, and (c) $6.6 \mathrm{GHz}$. The IMD in channel $k$ is found by calculating the output power at zero input power in channel $k$. The squares give the output signal and IMD when the phases in all the channels are equal. For the small channel spacing $(300 \mathrm{MHz})$, we observe an 8 -dB difference in the output signal level between the channels with the highest and the lowest carrier frequencies. This is due to the gain suppression effect [9], which also causes the highest relative IMD for the channel with the highest carrier frequency. The low channel spacing may be unrealistic for practical system applications, but it is
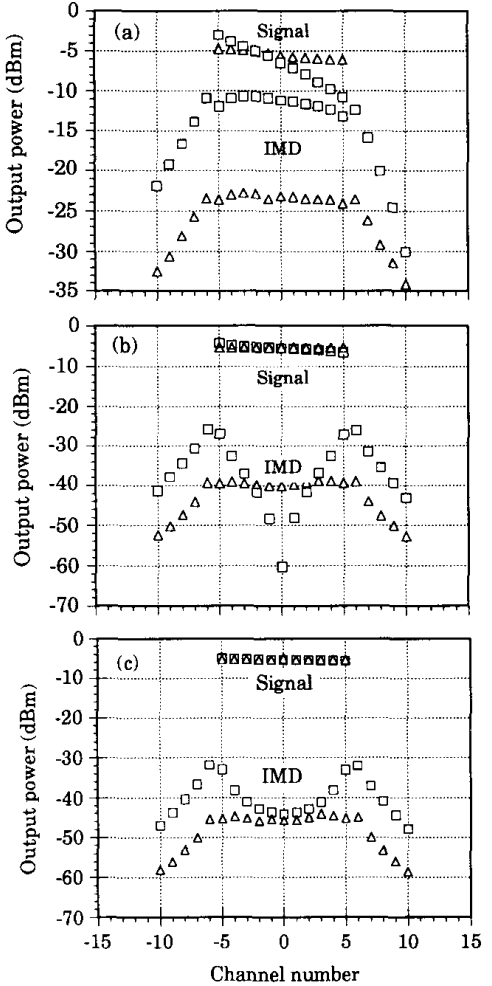

Fig. 11. Signal output power and IMD in 11 channels for channel spacings of (a) $300 \mathrm{MHz}$, (b) $3.3 \mathrm{GHz}$, and (c) $6.6 \mathrm{GHz}$. Gain: $25 \mathrm{~dB}$; input power: -30 $\mathrm{dBm} /$ channel; equal carrier phases: squares; random phases of the optical carriers; triangles.

included to clearly illustrate the excessive gain suppression. For a channel spacing of 3.3 and $6.6 \mathrm{GHz}$, the gain suppression causes a 3- and 1-dB signal level difference, respectively, while the center channel experiences a dip in IMD. This dip can be explained by the difference in the carrier-densityinduced phase shift $\left(\Phi=\arctan \left[2 \pi \Delta f \tau_{e}\right]\right)$, which causes IMD contributions from channels to the left- and the righthand side of the center channel to cancel out (i.e., $\Phi$ is $\sim-\pi / 2$ and $\sim \pi / 2$, respectively). In a real system, the phases will vary randomly resulting in a weaker modulation of the carrier density. The average levels for the IMD and the signal in each channel are also shown in Fig. 11 for the cases where random phases are included in the model. As seen, the IMD is reduced by as much as $13 \mathrm{~dB}$, and the gain suppression almost disappears. The results show the importance of including the random phase variation of the carriers when multichannel amplification is simulated.

\section{CONCLUSION}

A detailed model for multichannel amplification in semiconductor optical amplifiers has been developed. The model accounts for the carrier distribution in the longitudinal direction as well as the residual facet reflectivity. It offers the possibility to study multisection amplifiers operating with inhomogeneous current injection. 
Two-channel experiments with cascaded amplifiers showed good agreement between simulated and measured crosstalk and IMD. For 23-dB gain, an input power of $-20 \mathrm{dBm}$, and a channel spacing of $400 \mathrm{MHz}$, crosstalk and IMD levels of -12 and $-23 \mathrm{~dB}$ were measured after the first amplifier. The amplitudes of the crosstalk and the IMD contributed by each amplifier stage are added together. This means that, for multichannel systems with cascaded amplifiers, the accumulated IMD and crosstalk will increase more rapidly than the noise and may be the factors limiting the number of amplifiers. To ensure low crosstalk, FM modulation is preferable to AM modulation. Similarly, a low IMD is ensured by increasing the channel spacing.

The IMD can be reduced by incorporating an absorber section in the amplifier. Reductions of as much as $25 \mathrm{~dB}$ can be obtained for small channel spacings; however, for channel spacings larger than $300 \mathrm{MHz}$, no reduction is observed. Finally, for simulations of multichannel signals our investigation has clearly indicated that random phases of the optical carriers must be included in the model since this reduces the predicted IMD by up to $13 \mathrm{~dB}$.

\section{REFERENCES}

[1] J.C. Simon, "Semiconductor laser amplifier for single mode optical fiber communications," J. Opt. Commun., vol. 4, pp. 51-62, 1983.

[2] B. Fernier et al., "Design and realization of polarization insensitive semiconductor optical amplifiers with low forward current," in Proc. Topical Meeting Opt. Amplifiers and Their Applicat. (Monterey, CA), Aug. 1990 , pp. $82-85$

[3] A.J. Collar, "Low residual reflectivity of angled-facet semiconductor laser amplifiers," IEEE Photon. Technol. Lett., vol. 2, no. 8, Aug. 1990.

[4] B. Mikkelsen et al., "Temperature-dependent gain and noise of $1.5 \mathrm{\mu m}$ laser amplifiers," Electron. Lett., vol. 25, pp. 357-358, Mar. 1989.

[5] H.E. Lassen, P. B. Hansen, and K.E. Stubkjaer, "Crosstalk in $1.5 \mu \mathrm{m}$ InGaAsP optical amplifiers for coherent transmission systems," J. Lightwave Technol., vol. 6, pp. 1559-1565, Oct. 1988.

[6] T. Durhuus et al., "Crosstalk and intermodulation distortion in $1.5 \mu \mathrm{m}$ laser amplifiers," IOOC'89, Japan, 1989.

[7] N.G. Öberg and N.A. Olsson, "Crosstalk between intensity modulated wavelength division multiplexed signals in a semiconductor laser amplifier, IEEE J. Quantum Electron, vol 24, pp 52-59, Jan 1988.

[8] G. Grosskopf, R. Ludvig, and H.G. Weber, "Crosstalk in optical amplifiers for two channel transmisison," Electron. Lett., vol. 22, pp. 900-901, Aug. 1986.

[9] G.P. Agrawal, "Four-wave mixing and phase conjugation in semiconductor laser media," Opt. Lett., vol. 12, no. 4, Apr. 1987.

[10] R. M. Jopson, T.E. Darcie, and K. Gayliard, "Measurement of carrier density mediated intermodulation distortion in an optical amplifier," Electron. Lett., vol. 23, pp. 1394-1395, 1987.

[11] F. Favre, D. Le Guen, J.-C. Simon, and P. Doussière, "Four wave mixing in travelling wave semiconductor laser amplifier," Electron. Lett., vol. 25 , Feb. 1989

[12] G. Grosskopf, R. Ludvig, R. G. Waarts, and H. G. Weber, "Four wave mixing in a semiconductor laser amplifier," Electron. Lett., vol. 24 pp. 31-32, Jan. 1988 .

[13] T. Durhuus, "Intermodulation due to optical amplifiers in multichannel systems," in Proc ECOC'89 (Gothenburg, Sweden), 1989, vol. 1, pp. $46-49$.

[14] T. G. Hodgkinson and R.P. Webb, "Carrier-density modulation effects in a traveling wave semiconductor optical amplifier: Communications theory analysis and experiment," J. Lightwave Technol, vol, 9, no. 5, pp. 605-622, May 1991.

[15] B. S. Glance, G. Eisenstein, P. J. Fitzgerald, K.J. Pollock, and G. Raybon, "Crosstalk degradation caused by optical amplification in a multichannel FSK heterodyne system," J. Lightwave Technol., vol. 7 , no. 5, pp. 759-765, May 1989.

[16] G.P. Agrawal, "Amplifier induced crosstalk in multichannel coherent lightwave systems," Electron Lett., vol. 23, pp. 1175-1177, Oct. 1987.

[17] R.M. Jopson and T.E. Darcie, "Calculation of multicarrier intermodulation distortion in semiconductor optical amplifiers," Electron. Lett., vol. 24 , pp. $1372-1374$, Oct. 1988 .

[18] B. Tromborg, H. Olesen, X. Pan, and S. Saito, "Transmission line description of optical injection locking for Fabry-Perot and DFB lasers," I. Quantum Electron., vol. QE-23, pp. 1875-1889, Nov. 1987.

[19] M. J. Adams, J. V. Collins, and I. D. Henning, "Analysis of semiconductor laser optical amplifiers," Proc. Inst. Elec. Eng., vol. 132, pt. J, pp. $58-63$. Feb. 1985

[20] R. Ludwig, R. Schnabel, N. Schunk, and H. G. Weber, "Multisection semiconductor laser structures for optical traveling wave amplifiers," in Proc. Topical Meeting Opt. Amplifiers and Their Applicat. (Monterey, CA), Aug. 1990, pp. 86-89.

[21] H. Kawaguchi, "Optical bistable-switching operation in semiconductor lasers with inhomogenious excitation," Proc. Inst. Elec. Eng., vol. 129 pt .1, no. 4 , pp. 141-148, Aug. 1982 .

[22] I. D. Henning, M. J. Adams, and J. V. Collins, "Performance prediction from a new optical amplifier model," J. Quantum Electron., vol. QE-21, no. 6, pp. 609-613, June 1985 .

[23] R. Olshansky, C. B. Su, J. Manning, and W. Powazinik, "Measurement of radiative and nonradiative recombination rates in InGaAsP and AlGaAs light sources," IEEE J. Ouantum Electron., vol. QE-20, pp. 838-854, Aug. 1984

[24] G.P. Agrawal, "Evaluation of crosstalk penalty in multichannel ASK heterodyne optical communication systems," Electron. Lett., vol. 23 , pp. 906-908, Aug. 1987

[25] N. A. Olsson, "Lightwave systems with optical amplfiers," J. Lightwave Technol., vol. 7, pp. 1071-1082, July 1989.

[26] T. E. Darcie, R. M. Jopson, and R. W. Tkach, "Intermodulation distortion in optical amplifiers from carrier-density modulation," Electron. Lett. vol. 23 , pp. $1392-1394$, Dec. 1987.

[27] I. Cha, M. Kitamura, and I. Mito, " $1.5 \mu \mathrm{m}$ band travelling wave semiconductor optical amplifiers with window facet structure," Electron. Lett., vol. 25, pp. 242-243, Feb. 1989.

[28] A. A.M. Saleh, R.M. Jopson, and T.E. Darcie, "Compensation of noninearity in semiconductor optical amplfiers," Electron. Lett., vol. 24, pp. 950-952, July 1988 .

[29] L. D. Westbrook and M. J. Adams, "Simple expression for the linewidth enhancement factor in direct-gab semiconductors," Proc. Inst. Elec. Eng., vol. 134, no. 4, pp. 209-214, Aug. 1987

[30] N. Storkfelt, B. Mikkelsen, D.S. Olesen, M. Yamaguchi, and K. E Stubkjaer, "Measurement of carrier lifetime and linewidth enhancement factor for $1.5-\mathrm{mm}$ ridge-waveguide laser amplifier," IEEE Photon. Technol. Lett., vol. 3, pp. 632-634, July 1991.

[31] T. Durhuus, B. Mikkelsen, and K.E. Stubkjaer, "Influence of residual facet reflectivity on nonlinearities in semiconductor optical amplifiers," Electron. Lett., vol. 27, pp. 310-312, Feb. 1991

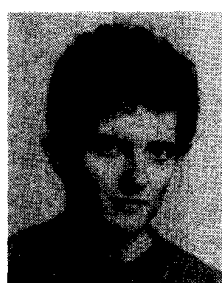

Terji Durhuus was born in Tórshavn, Faroe Islands, in 1962 . He received the M.Sc. degree from the Technical University of Denmark, Copenhagen, in 1989.

$\mathrm{He}$ is currently working as a research associate at the Electromagnetics Institute. His field of interest is optical amplifiers with emphasis on nonlinearities in semiconductor optical amplifiers.

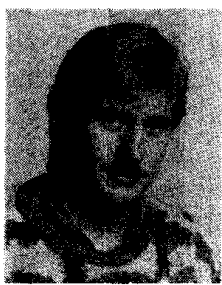

Benny Mikkelsen was born in $\mathrm{Sdr}$ Nissum, Denmark in 1960 . He received the M.Sc degree from the Technical University of Denmark (T.U.D.) Copenhagen, in 1987 . He is currently working toward the Ph.D. degree at the Electromagnetics Institute. His field of interest is optical amplifiers and their applications in optical networks. 


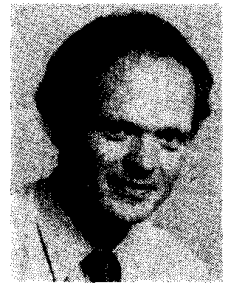

Kristian E. Stubkjaer ( $\left.S^{\prime} 80-M^{\prime} 81\right)$ was born in Denmark 1953. He received the M.Sc. and Ph.D. degrees from Technical University of Denmark, Copenhagen, in 1977 and 1981, respectively. From 1979 to 1981 he studied at the Tokyo Institute of Technology, Tokyo, Japan, with a scholarship form the Japanese Government.

From 1981 to 1982 he was drafted for military service at the Danish Defence Research Establishment in Copenhagen. From 1982 to 1983 he was a visiting scientist at the IBM T. J. Watson Research Center, Yorktown Heights, NY. He is now an Associate Professor at the Electromagnetics Institute, Technical University of Denmark, where he is working in the field of optical communication. From 1985 to 1990 he was Director of the Electromagnetics Institute and currently he is serving as chairman for the Electrotechnical Committee under the Technical Research Council (Danish Ministry for Education). 